e-mail submissions to correspondence@lancet.com

\title{
Plasma ascorbic acid and risk of heart disease and cancer
}

Sir-Kay-Tee Khaw and colleagues (March 3, p 657) ${ }^{1}$ report an inverse association between plasma ascorbic acid concentrations and mortality in men and women. They suggest that increased ascorbic acid intake might lessen risk of coronary heart disease and cancers. However, we think that the investigators have overinterpreted their results, since although adjustment was made for several risk factors, the potential major confounding of the chronic low-grade inflammatory response was not taken into account.

This omission is highly pertinent since evidence suggests that serum antioxidant concentrations (carotenoids, ascorbic acid) correlate strongly and inversely with markers of inflammation in illness and in apparently healthy individuals, ${ }^{2,3}$ and abundant data suggest that markers of low-grade inflammation predict vascular disease independently of traditional risk factors. ${ }^{4}$ Indeed, Khaw and Woodhouse ${ }^{5}$ have previously reported a significant inverse relation between markers of the acute-phase response (C-reactive protein and fibrinogen) and serum ascorbic acid concentrations in 96 men and women. Moreover, available evidence from studies in ill patients ${ }^{2}$ suggests that the observed low ascorbic acid concentrations do not alter with extra supplementation of this substance but rather by resolution of the inflammatory response.

The above observations could also explain the largely disappointing results of supplementation trials. Thus, we urge Khaw and colleagues to reanalyse their data with correction for a marker of the inflammatory response such as C-reactive protein (with a sensitive assay) or fibrinogen. Only with such analyses can we start to dissect the relevance of diet compared with inflammation in determining ascorbic acid concentrations and, by extrapolation, risk of vascular disease and cancers.

* Naveed Sattar, Donald McMillan

Departments of *Pathological Biochemistry and Surgery, Glasgow Royal Infirmary, Glasgow G31 2ER, UK

(e-mail: nsattar@clinmed.gla.ac.uk)
1 Khaw KT, Bingham S, Welch A, et al. Relation between plasma ascorbic acid and mortality in men and women in EPICNorfolk prospective study: a prospective population study. Lancet 2001; 357: 657-63.

2 Galloway P, McCMillan DC, Sattar N. The inflammatory response: relevance to biochemical nutritional monitoring of trace elements and vitamins. Ann Clin Biochem 2000; 37: 289-97.

3 Langlois M, Duprez D, Delanghe J, et al. Serum vitamin $\mathrm{C}$ concentration is low in peripheral arterial disease and is associated with inflammation and severity of atherosclerosis. Circulation 2001; 103: 1863-68.

4 Ridker PM, Cushman M, Stampfer MJ, Tracy RP, Hennekens CH. Inflammation, aspirin, and the risk of cardiovascular disease in apparently healthy men. $N$ Engl F Med 1997; 336: 973-79.

5 Khaw KT, Woodhouse P. Interrelation of vitamin $\mathrm{C}$, infection, haemostatic factors, and cardiovascular disease. BMf 1995; 310: 1559-63.

\section{Author's reply}

Sir-Naveed Sattar and Donald McMillan would like a reanalysis of the data with adjustment for a marker of the inflammatory response such as Creactive protein or fibrinogen. We did not have resources at the baseline survey to analyse these markers of inflammation in the cohort, but we plan to do these analyses in stored frozen samples.

Low-grade inflammation may indeed predict vascular disease independent of traditional risk factors, ${ }^{1}$ but we think this variable is not a confounder but a possible mechanism through which plasma ascorbic acid, or factors for which it is a surrogate marker, might alter disease risk. We have previously reported that plasma ascorbic acid relates inversely to markers of inflammation. ${ }^{2}$ Inflammation could lower plasma ascorbic acid concentrations; ascorbic acid, or factors for which it is a marker, could also modulate the inflammatory response. We have noted in the EPIC-Norfolk study and previous work ${ }^{2}$ that dietary sources are a major determinant of plasma ascorbic acid concentrations. Additionally, the magnitude of the relation between plasma ascorbic acid and cardiovascular disease (2-3-fold difference in risk between top and bottom quintiles of ascorbic acid) is larger than that reported for markers of inflammation. ${ }^{1}$
We entirely agree with the reservations about vitamin supplementation, which was why we went into some detail in our analyses, reporting no overall effect of supplement use and presenting dietary data. In our conclusions we were careful not to recommend ascorbic acid supplementation, but to state that our findings lend further support to the substantial existing evidence for the benefits of increasing intakes of fruit and vegetables, the major dietary sources of vitamin C.

\section{Kay-Tee Khaw}

Clinical Gerontology Unit Box 251, University of Cambridge, Addenbrooke's Hospital, Cambridge CB2 2QQ, UK

1 Danesh J, Collins R, Appleby P, Peto R. Association of fibrinogen, C-reactive protein, albumin, or leukocyte count with coronary heart disease. $7 A M A$ 1998; 279: 1477-82.

2 Khaw K-T, Woodhouse P. Interrelation of vitamin $\mathrm{C}$, infection, haemostatic factors and cardiovascular disease. BM7 1995; 310: 1559-63.

Sir-The EPIC-Norfolk prospective study, ${ }^{1}$ based on 96 deaths from ischaemic heart disease among men and 27 among women, showed an inverse relation between plasma ascorbic acid concentration and mortality. The relative risks for increasing plasma ascorbic acid concentration equivalent to an increase of $20 \mathrm{pmol} / \mathrm{L}$ of daily intake were 0.63 for men and 0.56 for women.

We analysed the relation between dietary intake of ascorbic acid and the risk of acute myocardial infarction (AMI) by use of data from a multicentre case-control study done in Italy between 1995 and 1999. The study included 507 patients (378 men, 129 women, median age 61 years [range 25-79]) who had non-fatal AMI, and 478 controls (297 men, 181 women, median age 59 years, [25-79]) admitted to hospital for acute disorders unrelated to smoking and other recognised risk factors for AMI. We used a validated food frequency questionnaire for 78 foods or food groups, and calculated intake of total energy, and selected macronutrients and micronutrients by use of an Italian food-composition database. ${ }^{2}$ The main 


\begin{tabular}{|c|c|c|c|}
\hline Ascorbic acid intake (mg/day) & AMI (n[\%]) & Controls (n[\%]) & Odds ratio $(95 \%) *$ \\
\hline$<81 \cdot 8$ & $124(24.5)$ & 95 (19.9) & $1.00 \dagger$ \\
\hline $81 \cdot 9-104 \cdot 7$ & $96(18.9)$ & $96(20 \cdot 1)$ & $0.69(0.44-1.07)$ \\
\hline $104 \cdot 8-133 \cdot 1$ & $118(23 \cdot 3)$ & $96(20 \cdot 1)$ & $0.81(0.52-1.25)$ \\
\hline $133 \cdot 2-184 \cdot 5$ & $92(18 \cdot 2)$ & $95(19 \cdot 9)$ & $0.73(0.46-1.16)$ \\
\hline$>184 \cdot 5$ & $77(15 \cdot 2)$ & $96(20 \cdot 1)$ & $0.55(0.34-0.89)$ \\
\hline Continuous & & & $0.86(0.74-1 \cdot 01)$ \\
\hline
\end{tabular}

$\mathrm{AMI}=$ acute myocardial infarction. *Estimates from multiple logistic regression equations for age, sex, education, physical activity, body-mass index, cholesterol, smoking, coffee, alcohol drinking, hyperlipidaemia, diabetes, hypertension, total energy intake, and family history of AMI in first-degree relatives. +Unit set as 1SD $(65.8 \mathrm{mg})$ distribution of controls.

\section{Dlstribution of 507 cases of acute myocardial infarction and 478 controls by dietary} ascorbic acid intake

sources of ascorbic acid in the Italian diet were citrus fruit (29\%), kiwi $(14 \%)$, tomatoes $(8 \%)$, apples, and pears, citrus fruit juice, and green and other salads (5-6\% each).

The multivariate odds ratios for subsequent quintiles of ascorbic acid intake are given in the table. There was an inverse association between ascorbic acid intake and AMI risk; the odds ratio for the highest quintile of intake compared with the lowest one was 0.55 , and the trend in risk was significant. When the unit was set to $1 \mathrm{SD}$ of the distribution of controls $(65.8 \mathrm{mg})$, the continuous odds ratio was $0 \cdot 86$. The inverse association was similar in strata of age at diagnosis of AMI, whereas it was stronger in current smokers (odds ratio 0.42 [95\% CI $0 \cdot 19-0.93$ for the highest quintile of intake) compared with non-smokers $(0.69[0.36-1 \cdot 30])$, and in people with a body-mass index $25.0 \mathrm{~kg} / \mathrm{m}^{2}(0.47$ [0.25-0.88]) compared with leaner people $(0 \cdot 83[0 \cdot 36-1 \cdot 88])$.

These findings further support the suggestion that the intake of vegetables and fruit rich in ascorbic acid is inversely related to the risk of AMI. Evidences on the relation between vegetables, fruit, and ascorbic acid intake and AMI are not consistent. In the Physicians' Health Study cohort, ${ }^{3}$ the relative risk for men consuming at least 2.5 servings per day of vegetables was $0 \cdot 77$. Among studies done in Mediterranean populations, in an Italian hospital-based case-control study, ${ }^{4}$ based on 287 women, the odds ratio of AMI for the highest tertile of intake compared with the lowest was 0.6 for fruit and 0.7 for green vegetables. However, in a hospitalbased case-control study done in Greece, ${ }^{5}$ including 329 cases of coronary heart disease of both sexes, researchers noted no association between ascorbic acid and coronary heart disease.

\section{*Alessandra Tavani, Carlo La Vecchia}

*Eva Negri, Michaela Bertuzzi, Istituto di Ricerche Farmacologiche "Mario Negri", via Eritrea 62, 20157 Milan, Italy; and Istituto di Statistica Medica e Biometria, Università degli Studi di Milano, Milan

(e-mail: tavani@marionegri.it)
1 Khaw K-T, Bingham S, Welch A, et al. Relation between plasma ascorbic acid and mortality in men and women in EPICNorfolk prospective study: a prospective population study. Lancet 2001; 357: 657-63.

2 Favero A, Salvini S, Russo A, et al. Sources of macro- and micronutrients in Italian women: results from a food frequency questionnaire for cancer studies. Eur $\mathcal{F}$ Cancer Prev 1997; 6: 277-87.

3 Liu S, Leel M, Ajani U, et al. Intake of vegetables rich in carotenoids and risk of coronary heart disease in men: the Physicians' Health Study. Int $\mathcal{F}$ Epidemiol 2001; 30: 130-35.

4 Gramenzi A, Gentile A, Fasoli M, et al. Association between certain foods and risk of acute myocardial infarction in women. BMF 1990; 300: 771-73;

5 Tzonou A, Kalandidi A, Trichopoulou A, et al. Diet and coronary heart disease: a case-control study in Athens, Greece. Epidemiology 1993; 4: 511-16.

Sir-The title of the study by Kay-Tee Khaw and colleagues ${ }^{1}$ and that of your March 3 acccompanying Talking Point exaggerate what the actual study states. My concern is that you do not fall into the same headline-grabbing mentality as much of the lay media.

In this particular case, I do not believe your headline that the story is consistent at all with what the study actually says, and that it is likely to mislead many consumers and professionals who do not take the time to review the actual study or have the educational background to do so.

Specifically, the following points could have been made. The results of the study show only an associated benefit of a higher intake of fruit and vegetables compared with a specific nutrient, which we already know. Higher plasma concentrations of ascorbic acid are not directly reflective of a direct relation with increased health benefits. As Jacobs ${ }^{2}$ and others have shown, the ascorbic acid concentration of the white blood cells is always much higher than that in plasma. There could be a striking drop in plasma ascorbic acid with only a moderate to minor drop in white blood cell concentration and immunity status. This difference holds true with all the purported antioxidants. For example, in a study by Meagher and colleagues, ${ }^{3}$ the participants were given $200,400,800$,
1200 , or 2000 IU daily for 8 weeks compared with placebo. Their plasma concentrations rose but the investigators questioned the rationale for vitamin-E supplementation in healthy individuals since the health benefits from the additional levels of vitamin $\mathrm{E}$ were no greater than without.

The average adult stores roughly $1200-1500 \mathrm{mg}$ ascorbic acid and uses around $3 \%$ of this amount per day. Adding one more serving of ascorbic acid ( $8 \mathrm{mg}$ to $65 \mathrm{mg}$ dependent on the food) would simply not explain the benefits obtained from the vitamin alone. The only explanation, which is far more consistent with the overall research, was well illustrated by researchers at Cornell University. ${ }^{4}$ The study's title was "Antioxidant activity of fresh apples". The report stated "the researchers found that the ascorbic acid in apples is only responsible for a small portion of the anti-oxidant activity. Instead, almost all of this activity in apples is from phytochemicals. Indeed, previous studies have shown that a $500 \mathrm{mg}$ ascorbic acid pill might be prooxidant. The Cornell researchers found that eating $100 \mathrm{~g}$ fresh apple with skins provided the total anti-oxidant activity equal to $1500 \mathrm{mg}$ ascorbic acid. What those findings show is that the combination of phytochemicals plays a very important role in anti-oxidant and anti-cancer activity, and the real health benefits may come from a phytochemical mixture." We know that $85 \%$ of the antioxidant activity of fruits and vegetables comes from chemicals other than the standard ascorbic acid, vitamin $\mathrm{E}$, and $\beta$-carotene, as the Cornell study illustrates.

This explanation is also likely for Khaw and colleagues' study, which should have been made more clear in the relevant titles.

David Lightsey

G\&G Physical Therapy Inc, 1201 23rd Street, Bakersfield, CA 93301, USA

1 Khaw K-T, Bingham S, Welch A, et al. Relation between plasma ascorbic acid and mortality in men and women in EPICNorfolk prospective study: a prospective population study. Lancet 2001; 357: 657-63.

2 Jacobs RA, Skala JH, Omaye ST. Biochemical indices of human vitamin $\mathrm{C}$ status. Am f Clin Nutr 1987; 46: 818-26.

3 Meagher EA, Barry OP, Lawson JA, Rokach J, Fitzgerald GA. Effects of vitamin $\mathrm{E}$ on lipid peroxidation in healthy persons. FAMA 2001; 285: 1178-82.

4 Eberhardt MV, Lee CY, Liu RH.

Antioxidant activity of fresh apples. Nature 2000; 405: 903-04.

Sir-Kay-Tee Khaw and colleagues ${ }^{1}$ show strong inverse associations between plasma ascorbic acid concentrations and mortality among people recruited from general practices 